\title{
Tasks Scheduling Using Dynamic Cluster-Based Hierarchical Real- Time Scheduler for Autonomous Car
}

\section{Girish Talmale*, Urmila Shrawankar \\ Department of Computer Science and Engineering, G H Raisoni College of Engineering, Nagpur}

Keywords: Cluster Scheduling, Real Time System.

\section{Abstract}

The recent advancement in information technologies makes autonomous cars in reality. The complexity of such cars increases day by day which results in high real-time computational demand. The existing multi-core real-time scheduling algorithms are based on partitioned and global scheduling approaches. The partitioned based algorithms having drawbacks like poor utilization bound, load balancing, not compatible with an open system environment and the global scheduling approach faces problems like high scheduling overhead. Cluster scheduling represents a hybrid scheduling approach that consists of a set of the processor as a cluster and tasks schedule to each processor of clusters using a global scheduling approach. The different task assignments heuristics are investigated for the homogeneous and heterogeneous cluster as well as a static and dynamic cluster that used the harmonic period aware technique. A new dynamic cluster-based hierarchical real-time scheduling algorithm for autonomous cars and compare the result with benchmarking algorithms in global and partitioned based real-time scheduling has been presented here. Experimentation performed on Simso multiprocessor realtime simulator.

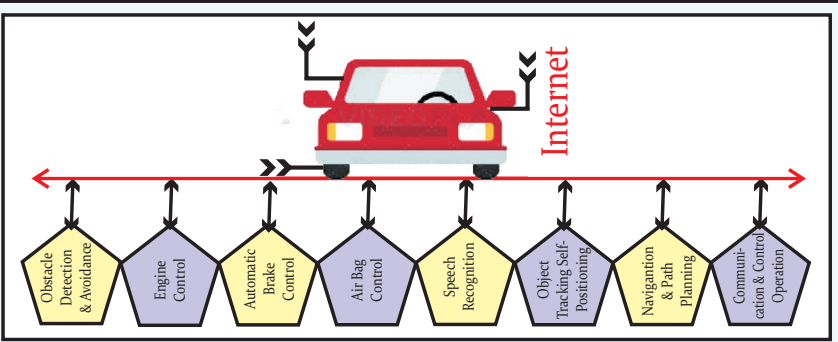

Figure.1 Real Time Operations in Autonomous Cars

speech recognition, automatic brake control system, engine control, airbag dashboard control and many more as shown in Fig.-1. The real-time computation the demand for such applications increase a lot and to satisfy that high computation demand real-time systems use multi-core as processing platform because of some drawbacks of uniprocessor processor like high power consumption, excessive heat dissipation, low computation over that multiprocessor offer several advantages like high and parallel computation, low power consumption per unit cost, low heat dissipation (Leng et al., 2020). Recent realtime software-industrial trends are to combine multiple

*Corresponding Author: girishtalmale@gmail.com 
real-time application and process it on a common multiprocessing platform to reduce cost and communication overheads (Agrawal et al., 2020). Real time Scheduling used to schedule different operations of Autonomous cars to provide safe, reliable and optimize working of Autonomous cars.

Autonomous Real-Time Scheduling Framework as shown in fig.-1 consists of different subsystems represented by clusters. Each cluster consists of multiple processors. The tasks of the subsystem are handled by processors allocated to the cluster.

Real-Time Scheduling of Multiprocessor System: the real-time multiprocessor scheduling problem is divided into two parts task assignment and scheduling. In task, assignment decides processor on which tasks gets executes. Scheduling problem decides task which tasks at what time will execute on processor i.e. priority of tasks . Task assignment problems are further divided into two types static and dynamic task assignments. In static tasks assignment which task executes on which processor determines statically. The advantages of static tasks assignment are all the task assignment is done prior so deadline of all tasks can be guaranteed. Dynamic real-time task assignment the tasks are assignment dynamically during run time. Literature study shows that an optimal solution for a dynamic real-time scheduler for more than one processor does not exist. This lead to the use of heuristic solutions to solve the problem (Martinez et al.,2020; Socci et al., 2019; Syed \& Fohler, 2019)

Hierarchical Real-Time Scheduling for Autonomous Cars: a smart embedded system such as avionic, satellite control, application of smart city, automotive system, and electrical grid composed of various subsystems. Recent trends in the real-time software industry combine multiple subsystems and processes on common processing platforms like multiprocessor systems to reduce communication and hardware cost. The global scheduler is responsible to schedule subsystem and the local scheduler in each subsystem is responsible for scheduling different subsystem tasks as shown in Fig.-2. Hierarchical real-time scheduling is a framework that provides a composition of multiple levels of subsystem hierarchically to provide temporal and fault isolation among the subsystems. Hierarchical scheduling framework for autonomous cars composed of subsystems such as obstacle detection subsystem consisting of two task obstacle detection and control, Subsystem that perform control tasks such as airbag control, automatic brake control and dashboard control consist of sensing and actuation tasks. Object tracking and self-positioning subsystem, navigation and planning, and engine control subsystem responsible to handle several tasks related to the operations of that subsystem.

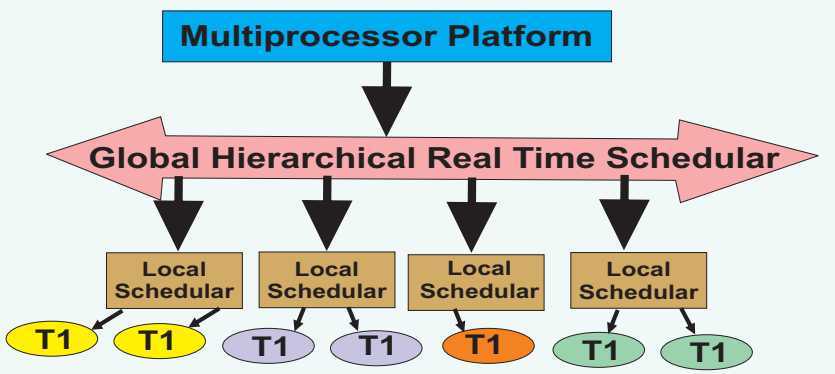

Figure-2: Hierarchical Real-Time Scheduling Framework (Concept: Agrawal et al., 2020).

Objectives of this technical study:

1. Develop cluster-based task assignment algorithm for Autonomous Cars

2. Present dynamic cluster-based hierarchical real-time scheduling algorithm for autonomous cars

3. Analysis of scheduling, migration and preemption overheads using a proposed scheduler

4. Comparative analysis of cluster, partitioned and global real-time scheduler.

\section{Methodology In Real-Time Scheduling :}

Real-time scheduling for multi-core is dividing into two main types global and partitioned based scheduling. Global and Partitioned based real-time scheduling has some pros and cons. Anderson et al., (2009) presented real-time scheduling for mixed critical systems such as autonomous cars. It uses Partitioned EDF for a set of tasks and Global EDF for a different set of tasks with different levels of tasks criticality. Bommert (2013) proposed a mixed critical real-time scheduling approach that offers an equal distribution of load with reduced overheads. Improvement schedules are introduced to get schedulability with the least asset use. The comparative analysis partitioned and global real-time scheduling for mixed critical systems and stated that partitioned based system performs better than global scheduling. Gomez et al., (2014) present a hierarchical scheduling approach that simplifies the partitioning of tasks. It makes the hierarchical composition of the real-time scheduler. Sigrist (2014) added more features in the hierarchical scheduling framework and reduced overheads. The above research shows that different subsystems require different real-time scheduling algorithms. There is a requirement for a hybrid scheduler that shows the feature of the partitioned and global real-time scheduler. The requirement of a scheduler with minimum scheduling overheads. Cluster-based scheduling is a hybrid scheduling approach that combines the features of global and partitioned scheduling (Hamdy et al., 2012). .

\section{Tasks and scheduling model:}

The real-time system with homogeneous multiprocessor consist of suppose n number of processors where $\mathrm{n}>\mathbf{1}$. 
Tasks Model: task model consists of a set of tasks that are periodic in nature represented by

$\mathrm{T}=\left\{\mathrm{t} 1, \mathrm{t} 2, \mathrm{t} 3^{-----\mathrm{tn}\}}\right.$ in Table-2. Each task is defined by its attribute like tasks arrival time (a), task ready time(r) by which task is ready to execute by one of the processors in the system and worst-case execution time of task(w). Each task generates the sequences of instances called jobs ( $\mathrm{Li} \&$ Cheng, 2015). The first job of each task is to assume to be generated at time zero i.e. tasks are set to be synchronous. Each task must finish its execution by the timeless than its period. The relative deadline of task (d) is nothing but the time interval between the start of time and the end of the execution of the tasks. Initially, tasks are considered to be non-primitive, non-periodic and independent (Davis et al., 2018; Hu \& Veeravalli, 2014).

\begin{tabular}{|c|c|}
\hline Task Parameter & Description \\
\hline $\mathrm{T}=\{\mathrm{t} 1, \mathrm{t} 2---------\mathrm{tn}\}$ & Set of Task in real time system \\
\hline Ti(ai,wi,pi,di) & $\begin{array}{l}\text { Task period ai as arrival time, wi } \\
\text { as worst case execution time } \\
\text { task Ti,pi as period of tasks, di } \\
\text { as relativedeadline }\end{array}$ \\
\hline Ji & $\begin{array}{l}\text { Represents instance of task Ti or } \\
\text { job of a TaskTi }\end{array}$ \\
\hline $\mathrm{Fi}$ & Finished time of Task Ti \\
\hline If $a i=o$ for all $T\{i=1,2-----n\}$ & Taskaresynchronous \\
\hline$a(i, j)=a(i, 1)+(j-1)^{*} p i$ & $\begin{array}{l}\text { Periodic task model where tasks } \\
\text { generate job instance after every } \\
\text { fix period }\end{array}$ \\
\hline $\mathrm{di}=\mathrm{pi}$ & Implicit deadline tasks model \\
\hline di $\neq$ pi & Constraint deadline tasks model \\
\hline $\begin{array}{l}\text { ui }=\text { wi } / \text { pi } \\
U=\sum \text { ui where } i=\left\{1,2^{-\cdots} \text { n }\right\}\end{array}$ & $\begin{array}{l}\text { Tasks utilization of } \mathrm{Ti} \\
\text { Total utilization of system }\end{array}$ \\
\hline
\end{tabular}

So no sharing of resources is required but the scheduling analysis will be able to implement resource synchronization protocols like priority ceiling protocol and stack resource policy (Bertogna et al., 2009). Task job represents the small execution unit of a task that cannot run on multiple processors (Salehi et al., 2015). Tasks can use some resources other than processors like data structure, memory input and output devices in shared or exclusive mode presented by $\mathrm{U}=\mathrm{W} / \mathrm{T}$ (worst- case execution time/Period of task) (Behnam et al., 2010). All tasks are assumed to be in non- shareable mode (Table-1).

Dynamic clustered Real-Time scheduler for autonomous cars :

It include two main phases: Task Assignment: In task assignment, the set of tasks assigned to cluster for execution (Qi et al., 2010). Tasks are sorted according to their harmonic periods and allocate to cluster to reduce scheduling overheads (Kim et al., 2014). If tasks are not harmonic then separate tasks with smaller periods from ones with larger periods and allocate them to different clusters to reduce scheduling overheads (Gonzalez et al., 2017; Zhong \& Gaochao, 2012).

Table -2: System Model Parameter

\begin{tabular}{|c|c|}
\hline Task Parameter & Description \\
\hline $\mathrm{P}=\left\{\mathrm{P}_{1}, \mathrm{P}_{2}, \mathrm{P}_{3}-{ }^{--------\mathrm{Pn}}\right\}$ & $\begin{array}{l}\text { Set of processors in multi-core } \\
\text { real timesystem }\end{array}$ \\
\hline CSIZE & $\begin{array}{l}\text { Defines number of clusters in } \\
\text { system CSIZE }=1 \text { for global } \\
\text { scheduling and CSIZE }=\mathrm{n} \text { i.e } \\
\text { number of processors in the } \\
\text { System for Partitioned based } \\
\text { scheduling }\end{array}$ \\
\hline $\mathrm{C}=\{\mathrm{c} 1, \mathrm{c} 2-----\mathrm{c}[\mathrm{CSIZE}]\}$ & Define set of cluster in system \\
\hline $\mathrm{M}=\{\mathrm{m} 1, \mathrm{~m} 2,------\mathrm{m}[\mathrm{CIZE}]\}$ & $\begin{array}{l}\text { Define number of processors in } \\
\text { each Cluster e.g mı represents } \\
\text { numberof Processors incluster c1 }\end{array}$ \\
\hline $\mathrm{Mi}=\mathrm{n} / \mathrm{CSIZE}$ & $\begin{array}{l}\text { For all cluster homogeneous } \\
\text { cluster having equal no of } \\
\text { Processorsinallclusters }\end{array}$ \\
\hline
\end{tabular}

Task assignment in Dynamic Cluster Real-Time scheduler forAutonomous Cars:

Assumption: $i$ is the index of tasks with ai as minimum arrival time, ci as worst-case execution time, pi as period and di as the relative deadline of task $\mathrm{i}$

Task set $\mathrm{T}=\{\mathrm{t} 1, \mathrm{t} 2, \mathrm{t} 3,--------\mathrm{tn}\}$

Let system consist of $\mathrm{x}$ no of processors $\left\{\mathrm{P}_{1}, \mathrm{P}_{2}, \mathrm{P}_{3}------\mathrm{Px}\right\}$ c represents the size of clusters No of clusters are $\mathrm{C}_{1}, \mathrm{C}_{2}$---$\mathrm{Cn}$ and each contains $\mathrm{x} / \mathrm{c}$ no of processors

$\mathrm{U}(\mathrm{x})$ represent utilization bound of $\mathrm{x}$ processor (Table-2)

Tasks sorted in ascending orders of their period

Tasks are independent of each other and having implicit deadlines i.epi=di

Each task can produce a number of task instances called jobs and the first instance of all tasks arrived at time o means tasks are synchronous. Tasks cannot execute in parallel on multiple processors at a time

\section{Algorithm:}

1. Set $\mathrm{pm}=$ maximum period of tasks

2. Initialize the queue with ordered task list TQUEUE $=\{\mathrm{F}\}$

3. while (Task Set $T \neq 0$ ) do

4. Find a task ti having a minimum task period

5. Put task ti in Harmonic Task Set $\mathrm{H}=\{\mathrm{ti}\}$

6. Remove task ti from Tasks Sets $\mathrm{T}=\mathrm{T}-\{\mathrm{ti}\}$

7. Initialize $\mathrm{L}=\mathrm{pi}\{$ period of task $\mathrm{ti}\}, \mathrm{k}=\mathbf{1}$

8. while( $\mathrm{L} . \mathrm{k}<=\mathrm{pm})$ do

9. if $\mathrm{tx}$ belongs to Task Set $\mathrm{T}$ and $\mathrm{px}=\mathrm{L} . \mathrm{k}$

10. for all such $t x$ with $\mathrm{px}=\mathrm{L} . \mathrm{k}$

11. $\mathrm{H}=\mathrm{H}+\{\mathrm{tx}\}$

12. Task Set $\mathrm{T}=\mathrm{T}-\{\mathrm{tx}\}$

13. End for

14. $\mathrm{L}=\mathrm{L} . \mathrm{k}$

15. $\mathrm{k}=1$ 
16. else

17. $\mathrm{k}=\mathrm{k}+\mathrm{1}$

18. End if

19. End while

20. Move all tasks in $\mathrm{H}$ set in increasing period order to TQUEUE

21. End while

22. Initialize cluster size based on the above heuristics

23. Assign tasks in TQUEUE to cluster

24. Assign the task to the processor of cluster using dynamic task assignment

25. End

The efficiency of cluster real-time multiprocessor scheduling is depending on task assignment strategies (Dairi et al., 2018). The tasks assigned to the cluster is schedulable only when the sum of the utilization of these tasks must be less than the total processor in the cluster (Davis \& Burne, 2011). The utilization bound of the cluster is used to effectively check the schedulability of task sets. The tasks sets assign to the cluster is schedulable only when the total utilization of the task is less than the utilization bound of the cluster. The proposed task assignment algorithm is based on the period of the tasks. The period of the task is also responsible for the security and performance of the schedulability test. The period based assignment also reduces the complexity of the scheduler and worst-case execution time of tasks. The tasks are sorted based on their harmonic period (Zhuravlev et al.,2011). The set of tasks are said to be harmonically related. If tasks sets are harmonically related then even for highly utilizing tasks it is possible to have a linear time schedulability test and response time analysis can be done in polynomial time (Ali \& Kim, 2017). The fault Tolerance mechanism for fault detection and recovery can be efficiently implemented if the period of tasks is harmonically related. The harmonic period is used in much industrial real-time applications (Xie \& Qin., 2008).

Dynamic Cluster Task Scheduling in Autonomous Cars: The tasks assignment is done, scheduling decides the order of execution of tasks on processors. Fairness implies that every task gets processor share proportional to its utilization factor. P-fair global scheduling having drawbacks of more scheduling overheads, migration and context switches. The dynamic cluster-based hierarchical real-time scheduler makes scheduling decisions of tasks only at tasks period boundaries instead of at every time slot like P-Fair global scheduling. In the Task scheduling phase, the tasks are scheduled to cluster processors based on task priority. The tasks are divided into task slots. Tasks slots are integral multiple of task period.

The dynamic cluster-based hierarchical real-time scheduling algorithm is as follows:

1. Forall tasks sets of cluster $\mathrm{C}_{1}$ to $\mathrm{Cn}$

2. Create task slots $\{$ Tbo,Tb1-----------Tbn $\}$ where Tbo $=0$ and
Tbn=LCM of all tasks periods

3. Tbi $=X^{*}$ Pi where $X=\left\{1,2,3^{--}\right\}$and $P i$ is the period of Task Ti

4. Forall taskstı to tn do

5. Calculate remaining work of tasks

a. Rwix $=w^{*}{ }^{*} \mathrm{Tbx}-$ allocated units for Ti, Wi=ci/pi

6. Calculate mandatory unit of tasks to ensure proportionate fairness

a. $\operatorname{Mik}=\max \left\{\mathrm{o},\left\{\left(\mathrm{RWik}+\mathrm{wi}^{*}(\mathrm{Tbx}+1-\mathrm{Tbx})\right\}\right\}\right.$

7. Calculate extra unit which is total time subtracted from mandatory unit and utilization of the cluster.

8. Extra Unit=Total Processor in Cluster $(\mathrm{Tbx}+1-\mathrm{Tbx})$ - Sum of all mandatory units of tasks in the cluster

9. Forall tasks ti to tn

10. Find tasks having pending work and not fullyallocated

11. Pwik=Rwik + wi* (Tbx+1-Tbx $)-\operatorname{mix}>0$.

12. Go to line 7 to select the next task

13. For all tasks having pending work and not fully allocated

14. Dynamically calculate the priority of tasks based on current task utilization afterallocation of mandatory unit

15. if multiple tasks having same utilization then decide priority based on a first come first serve basis

16. Forall high priority tasks do

17. Allocate each extra unit to high priority tasks

18. Eik $+1=1$ for time slot $\mathrm{k}+1$

19. End for

20. Forall tasks Ti to Tn do

21. Calculate the Remaining time of tasks for time slot $k+1$

22. Rwix $=$ Pwik+1 - Eik+1

23. End for

24. Generate schedule for $\{\mathrm{Tbx}, \mathrm{Tbx}+1\}$

25. Select next time boundary $\{\mathrm{Tbk}+1, \mathrm{Tbk}+2\}$

26. Select Next Cluster

27. End

\section{Resultsand Discussion:}

Experiments are performed using a real-time scheduling simulator. Simulator measures the different scheduling overheads, preemption costs, migration costs. Simulation performs on a different set of tasks sets with variable sizes of clusters.

Comparison of Cluster Scheduling with Global Scheduling and Partitioned Scheduling: the cluster scheduling solves the problem of partitioned as well as global scheduling. In cluster scheduling tasks are allocated to a group of processors called clusters than the sets of tasks assigned to processors of the cluster by using global scheduling. In each cluster, separate global scheduling with full utilization is used. Cluster-based global scheduling algorithms are based on continuous and discrete-time models. A continuous time based global scheduling algorithm can make scheduling decisions at any instant point. Discrete time based global scheduling algorithm once the tasks are assigned to the processor it cannot be executed for less than one-time unit. The discrete time-based scheduler PFair (Proportionate Fair) and its types can schedule tasks sets with full utilization but having drawbacks like high scheduling overheads, tasks migration and preemption overheads. The dynamic 
cluster-based scheduler is also a discrete-time modelbased global real-time scheduler for multiprocessors. it provides full utilization and reduces scheduling overheads as well as task migration and preemption overheads. The below example shows how cluster-based scheduling algorithms reduce scheduling overheads, migration and preemption overheads. The task set consists of six tasks with worst-case execution time and period of the task. The reduction in tasks migration and preemption significantly reduce the scheduling overheads of cluster-based

$\mathrm{T}=\left\{\mathrm{t}_{1}(1,3), \mathrm{t}_{2}(2,5), \mathrm{t}_{3}(2,7), \mathrm{t} 4(3,10), \mathrm{t}_{5}(2,7), \mathrm{t} 6(3,12), \mathrm{t}_{7}(2,8), \mathrm{t} 8\right.$ $(1,5), \mathrm{t} 9(2,15), \mathrm{t} 10(2,10), \mathrm{t} 11(3,9), \mathrm{t} 12(3,12)\}$

$\mathrm{L}$ as the Longest common multiplier is 30 . The clusterbased tasks allocation run on the above set of task with implicit deadline where the period of tasks is equal to the deadline of tasks. The result is as shown in the table which assigns each task to its core index and cluster index. Tasks can migrateamong the core index of the same cluster.

No of the processor in system $=04$ and No of cluster $=02$, Cluster $\mathrm{C}_{1}$ contain $\mathrm{P}_{1}$ and $\mathrm{P}_{2}$ Processor. Cluster $\mathrm{C}_{2}$ contain $\mathrm{P}_{3}$ and $\mathrm{P}_{4}$ Processor. Find the task ti with the minimum task period which is t1. Find tasks whose periods are multiple of taskı period and place all the tasks in harmonic set $\mathrm{H}$ and remove tasks from $\mathrm{T}$

$\mathrm{H}=\{\mathrm{t} 1(1,3), \mathrm{t} 6(3,12), \mathrm{t} 9(2,15), \mathrm{t} 11(3,9), \mathrm{t} 12(3,12)\}$

$\mathrm{T}=\left\{\mathrm{t}_{2}(2,5), \mathrm{t}_{3}(2,7), \mathrm{t} 4(3,10), \mathrm{t} 5(2,7), \mathrm{t} 7(2,8), \mathrm{t} 8(1,5), \mathrm{t} 10(2,10)\right\}$

Select task from T which having minimum period So tasks t $2(2,5)$ selected. Find out tasks having period multiple of task 2 period Which is $4(3,10), \mathrm{t} 8(1,5), \mathrm{t} 10(2,10)$ soadd all these tasks including task 2 in harmonic set and remove from T So as to get following result.

$\mathrm{H}=\{\mathrm{t} 1(1,3), \mathrm{t} 6(3,12), \mathrm{t} 9(2,15), \mathrm{t} 11(3,9), \mathrm{t} 12(3,12), \mathrm{t} 2(2,5), \mathrm{t} 4(3,10), \mathrm{t} 8(1,5$ )$, \mathrm{t} 10(2,10)\}$ and

$\mathrm{T}=\left\{\mathrm{t}_{3}(2,7), \mathrm{t}_{5}(2,7), \mathrm{t}_{7}(2,8)\right\}$

Find out tasks having minimum period from $\mathrm{T}$ So task $\mathrm{t}_{3}$ having a minimum period. Select all tasks from T including $\mathrm{T}_{3}$ having a period equal to $t_{3}$ and multiple of tasks 3 period Which is $\mathrm{t}_{3}(2,7)$ and $\mathrm{t}_{5}(2,7)$ so add these tasks to $\mathrm{H}$ and remove from

$\mathrm{TH}=\{\mathrm{t} 1(1,3), \mathrm{t} 6(3,12), \mathrm{t} 9(2,15), \mathrm{t} 11(3,9), \mathrm{t} 12(3,12), \mathrm{t} 2(2,5), \mathrm{t} 4(3,10), \mathrm{t} 8(1$ ,5), t10 $\left.(2,10), \mathrm{t}_{3}(2,7), \mathrm{t} 5(2,7)\right\}$ and

$\mathrm{T}=\left\{\mathrm{t}_{7}(2,8)\right\}$

Find out tasks having minimum period from $\mathrm{T}$ So task $\mathrm{t} 7$ having a minimum period. Select all tasks from $\mathrm{T}$ including $\mathrm{T}_{7}$ having a period equal to 77 and multiple of tasks 7 periods, which is $\mathrm{t}_{7}(2,8)$, So add these tasks to $\mathrm{H}$ and remove them from $\mathrm{T}$

$\mathrm{H}=\left\{\mathrm{t}_{1}(1,3), \mathrm{t} 6(3,12), \mathrm{t} 9(2,15), \mathrm{t} 11(3,9), \mathrm{t} 12(3,12), \mathrm{t} 2(2,5), \mathrm{t} 4(3,10), \mathrm{t} 8(1,5\right.$ $\mathrm{T}=\{\}$ )$\left., \mathrm{t} 10(2,10), \mathrm{t}_{3}(2,7), \mathrm{t} 5(2,7), \mathrm{t}(2,8)\right\}$ and

$\mathrm{H}=\{\mathrm{t} 1(1,3), \mathrm{t} 6(3,12), \mathrm{t} 9(2,15), \mathrm{t} 11(3,9), \mathrm{t} 12(3,12), \mathrm{t} 2(2,5), \mathrm{t} 4(3,10), \mathrm{t} 8(1,5$ )$\left., \mathrm{t}_{10}(2,10), \mathrm{t}_{3}(2,7), \mathrm{t}_{5}(2,7), \mathrm{t}(2,8)\right\}$ and

Allocate all the tasks from $\mathrm{H}$ to cluster with total utilization less or equal to a number of processors in the cluster. So allocate tasks t1 $(1,3), \mathrm{t} 6(3,12), \mathrm{t} 9(2,15), \mathrm{t} 11(3,9), \mathrm{t} 12(3,12), \mathrm{t} 2(2,5)$ to cluster $\mathrm{C}_{1}$ as utilization of these tasks is 1.96 less than 2 (no of processor).

A 11 o c a e $\mathrm{re} \mathrm{m}$ a i n i n g t a s k $\mathrm{t}_{4}(3,10), \mathrm{t} 8(1,5), \mathrm{t} 10(2,10), \mathrm{t}_{3}(2,7), \mathrm{t}_{5}(2,7), \mathrm{t}_{7}(2,8)$ to cluster $\mathrm{C}_{2}$ as their total utilization is $1.52<2$ (no of processors in $C_{2}$ ). Once the tasks allocation is done now use a cluster scheduler that decides when and in what order these tasks will allocate to cluster processors. Dynamic cluster-based real-time scheduler schedules all tasks with $100 \%$ CPU utilization which is not possiblewith partitioned based real-timescheduling (Fig.-3).

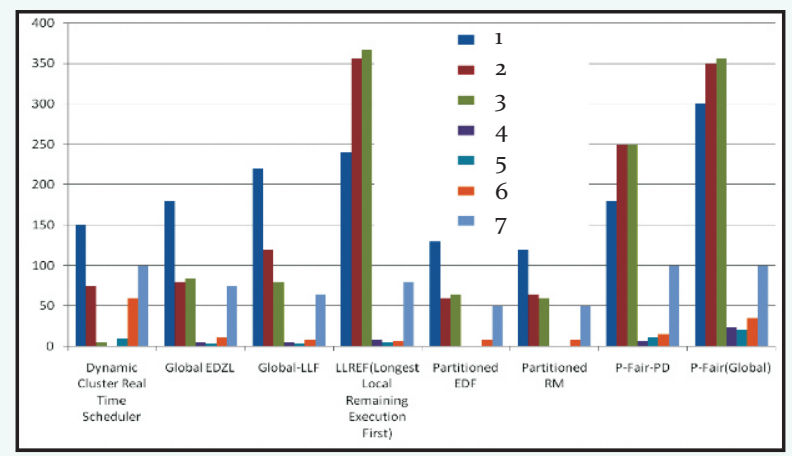

Figure 3 : Comparison of Dynamic Cluster Scheduler with Partitioned and Global Scheduler

1-Sum of Scheduling Count, 2- Sum of Processor context save count; 3Sum of Processor context Load count, 4- Sum of Average Preemption cost of Task; 5- Sum of Average Migration cost Task; 6- Sum of Average Response Time of Task; 8-Sum of CPU Utilization (\%)

\section{Conclusion:}

This paper described the dynamic clustered based realtime scheduling algorithms for Autonomous Cars, where the tasks are executed on a group of processors called clusters of the processor which solves the problem of partitioned scheduling, improve processor utilization. It also solves the problem of global scheduling of real-time systems with reduced job migration overhead, preemption overhead, management of data structure of tasks. The performance of cluster-based scheduling depends upon the size of the cluster, number of processors in the system, number of processors in each cluster, task assignment heuristics and the scheduling techniques. Cluster scheduling is a hybrid scheduling approach that can be used as partitioned and global scheduling both depends upon the set of task sets. Dynamic clustered realtime scheduling solves the problem of semi-partitioned real-time scheduling algorithms by the online splitting of tasks into clusters. The analysis of the proposed scheme is done through the Simso simulator and the simulator results shows that the number of context switches is small as compared to the global scheduling approach and as the size of the cluster gets smaller, the number of context switches also get smaller. The utilization bound analysis on dynamic cluster scheduling shows that the number of clusters is inversely proportional to utilization bound and directly proportional to maximum utilization of tasks means as soon as the number of clusters and maximum utilization of tasks increases, the utilization bound of cluster scheduling decreases. The number of tasks migration is also reduced in cluster scheduling as compared to the global scheduling approach and when the size of the cluster gets smaller the number of tasks 
migration also get reduced. The system utilization and successes ratio also improved as compare to partition scheduling. The Dynamic cluster-based real-time scheduler optimizes the performance of Autonomous cars as it helps to reduce tasks communication, preemption and migration overheads. Dynamic clustered real-time scheduling can be applied to a heterogeneous cluster multiprocessor systems in future work.

\section{References:}

Anderson, J., Baruah, S. \& Brandenburg, B. (2009): Multicore operating -system support for mixed-criticality. Proceedings of the Workshop on Mixed Criticality: Roadmap to Evolving UAV Certification

Agrawal, K., Baruah, S. \& Ekberg, P. (2020): Optimal scheduling of measurement-based parallel real-time tasks. Real-Time Syst. 56:247-253.

Ali, A. \& Kim, K. (2017): Cluster Based Multicore Real Time Mixcriticality Scheduling.J. Sys. Architect., 79:45-58.

Behnam, M., Thomas, N., Sjödin, M. \& Shin, I. (2010): Over-run Methods and Resource Holding Times for Hierarchical Scheduling of Semi-Independent Real-Time Systems. IEEE Transact. Indus. Inform., 6(1).

Bertogna, M., Fisher, N. \& Baruah, S. (2009): Resource-Sharing Servers for Open Environments. IEEE Transact. Indus. Inform., 5(3). Biondi, A., Buttazzo, C. \& Bertogna, M. (2015): Schedulability analysis of hierarchical real-time systems under shared resources. IEEE Transact. Comp., 65(5):15931605 .

Bommert, M. (2013): Schedule-aware distributed of parallel load in a mixed criticality environment. Proc. JRWRTC, RTNS., 21-24

Dairi, A., Harrou, F., Senouci, M., \& Sun, Y. (2018) : Unsupervised obstacle detection in driving environment using deep learning based stereovision. Robot Auton. Sys., 100:287-301.

Davis, R., Altmeyer, S. \& Reineke, J. (2018): Response-time analysis for fixed-priority systems with a write-back cache. Real-Time Syst., 54:912-963.

Davis, R. \& Burne, A. (2011): Survey of Hard Real-Time Scheduling for Multiprocessor Systems. Comp. Surv. ACM, 35:44.

Gomez, A., Schor, L., Kumar, P. \& Thiele, L. (2014): SF3P: a framework to explore and prototype hierarchical compositions of real-time schedulers. 201425 nd IEEE Int. Symp. Rapid Syst. Prototyp, 2-8 pp.

Gonzalez, A., Vazquez, D., Lopez, M. \& Amores, J. (2017): On Board Object Detection ,Multicure Multimodel,and Multiview Random Forest of Local Expert. IEEE Trans. Cybern., 47(11):3980-3990.
Hamdy, A., Reda, A. \& Youssef, A. (2012): Efficient scheduling of Real Time parallel application on clusters. Int. J. Comp. Sci. Info. Technol.,4(5):197-212.

Hu, M. \& Veeravalli, B. (2014): Dynamic scheduling of hybrid Real-Time tasks on clusters. IEEE Trans. Comp., 63(12):29882997.

Kim, J., Kim, D., Jang, C., \& Sunwoo, M. (2014): Development of autonomous car Part-1 distributed system architecture and development processes. IEEE Transact. Electron., 61(12): 7131-7140.

Leng, C., Qiao, Y., \& Hu, X. (2020): Co-scheduling aperiodic realtime tasks with end-to-end firm and soft deadlines in twostage systems. Real-Time Syst., 56:391-451.

Li, Y. \& Cheng, A. (2015): Transparent Real-Time task scheduling on temporal resource partitions. IEEE Trans. Comp. 65(5): 1646-1655,

Martinez, J., Sañudo, I. \& Bertogna, M.(2020): End-to-end latency characterization of task communication models for automotive systems. Real-Time Syst., 56:315-347.

Qi, X., Zhu, D. \& Aydin, H.(2010): A study of utilization bound and Run-Time overhead for cluster scheduling in multiprocessor real-time systems. In: The Sixteenth IEEE International Conference on Embedded and Real-Time Computing Systems and Applications. 3-12.

Salehi, M., Ejlali, A. \& Bashir M. (2015): Two-phase low-energy Nmodular redundancy for hard real-time multi-Core Systems. IEEE Trans. Parall. Distribut. Syst., 27(5):1497-1510.

Sigrist, L. (2014): Implementation and evaluation of mixedcriticality scheduling algorithms for multi-core systems. Technical Report SA-2013-57, Swiss Federal Institute of TechnologyZurich

Socci, D., Poplavko, P. \& Bensalem, S. (2019): Priority-based scheduling of mixed-critical jobs. Real-Time Syst., 55: 709-773.

Syed, A. \& Fohler, G.(2019): Efficient offline scheduling of task-sets with complex constraints on large distributed time-triggered systems. Real-Time Syst., 55:209-247.

Xie, T. \& Qin, X. (2008): Security aware resourse allocation for Real-Time parallel jobs on homogeneous and heterogeneous clusters. IEEE Trans. Parallel Distrib. Syst., 19(5):682-697.

Zhong, X. \& Gaochao, G. (2012): A task scheduling algorithm for multi-core cluster system. J. Comp., 7(11):2797-2804.

Zhuravlev, S. Carlos, J. \& Prieto, M. (2011): Survey of scheduling techniques for addressing shared resources in multicore processors. ACM Comp. Surv., 5:1-31. 